\title{
Properties of a Useful Biorthogonal System*
}

\author{
L. V. Spencer and P. Flusser**
}

(July 17, 1967)

\begin{abstract}
In radiation penetration theory, infinite medium flux distributions have for some years been calculated using biorthogonal functions called $U_{n}^{k}(z)$. In this paper the spaces spanned by these functions, transformation kernels, generating functions, recursion relations, asymptotic trends for large $n$, many expansions, and relations with well-known orthogonal polynomials are worked out.
\end{abstract}

Key Words: Biorthogonal functions, gamma-ray penetration theory, neutron penetration theory, polynomial approximations.

\section{Introduction}

The biorthogonal (BON) system discussed in this paper was encountered in 1950 during attempts to construct spatial distributions of the gamma-ray flux from the values of the first few even moments. The desired distributions appeared to depend on $|z|$, rather than $z^{2}$, so that it did not seem reasonable to represent the distributions with polynomials which utilized powers of $z^{2}$, as would have been the case for an orthogonal polynomial representation. In discussing this basic asymmetry with Spencer, U. Fano suggested that perhaps one should approximate with functions whose Laplace transforms were even functions of the transform parameter, but otherwise with the right kind of singularity, properly located.

This suggestion led to study of a set of functions which quickly were shown to have biorthogonality properties; and their use has made possible the calculation of large quantities of radiation data [1-2]. ${ }^{1}$ Reference 3 contains a brief discussion of this and related BON systems, together with some elementary properties easily derived, including the differential equations (3d order) satisfied by the functions. In this connection, it has since been proven by S. Preiser that the relevant differential equations are the only $3 \mathrm{~d}$ order differential equations whose solutions are polynomials and have the biorthogonality property [4].

During the past two years, further properties of these sets of functions have been studied; and this manuscript summarizes the most important results. The investigations have been extended into the approximation theory appropriate to these biorthogonal sets, and beyond this, some results have been obtained for related ("function fitting") types of approximations which have been extensively used in calculations of radiation data and other applications $[5,6]$.

The work has been largely stimulated by the intrinsic beauty of the mathematics, although the practical and theoretical importance of these sets of functions is considerable. And because there appears to be a lack of practical information about biorthogonal functions, a corresponding effort has been made to be reasonably complete in this presentation.

\section{General Structure}

$B O N$ "Bridges." BON systems such as those on which our attention is focused, are derivable from, and relatable to well-known orthogonal systems. To exhibit this clearly, we make use of

\footnotetext{
*An invited paper. Work supported by the Office of Civil Defense and the Defense Atomic Support Agency.

*An invited paper. Work supported by the Office of Civil Defer
**Present address, Ottawa University, Ottawa, Kansas 66067.

${ }^{1}$ Figures in brackets indicate the literature references at the end of this paper.
} 
mathematical structures called pairings [7]. A pairing is an ordered triple $(W, \hat{W},\langle\rangle$,$) , with W$ and $\hat{W}$ vector spaces over the same field $F$, and $\langle$,$\rangle a functional on W \times \hat{W}$ which is linear in the first coordinate and antilinear in the second. The functional $\langle$,$\rangle is also frequently referred to as a$ pairing.

Let $(W, \hat{W},\langle\rangle$,$) and (\Omega, \hat{\Omega},[]$,$) be two pairings. A linear transformation, \hat{B}: \hat{W} \rightarrow \hat{\Omega}$, will be called adjoint if there exists another transformation, $B: \Omega \rightarrow W$ such that

$$
[\pi, \hat{B} \hat{p}]=\langle B \pi, \hat{p}\rangle,
$$

for all functions $\pi \epsilon \Omega$ and $\hat{p} \epsilon \hat{W}$. A linear transformation. $\hat{\Theta}: \hat{\Omega} \rightarrow \Omega$, will be called self-adjoint if and only if

$$
[\Theta \hat{\sigma}, \hat{\tau}]=[\hat{\theta} \hat{\tau}, \hat{\sigma}]^{* 2}
$$

for every pair of elements, $\hat{\sigma}, \hat{\tau}$, in $\hat{\Omega}$.

In what follows we assume that in addition to the two pairings mentioned, $(W, \hat{W},\langle\rangle$,$) and$ $(\Omega, \hat{\Omega},[]$,$) , we have a linear transformation, B: \Omega \rightarrow W$, its adjoint linear transformation, $\hat{B}: \hat{W} \rightarrow \hat{\Omega}$, and a self-adjoint transformation, $\Theta: \hat{\Omega} \rightarrow \Omega$. We define

$$
T=B \Theta \hat{B} .
$$

The situation is illustrated in figure 1 .

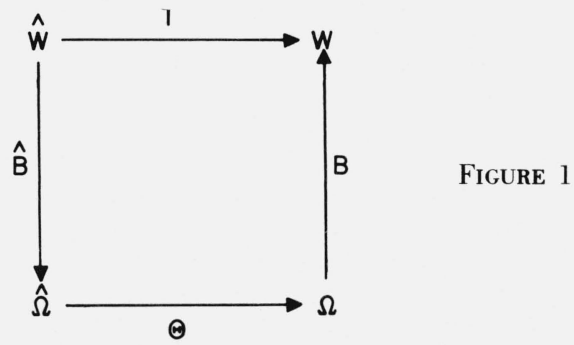

Clearly, if $\Theta$ is self-adjoint, so is $T$. For let $\hat{s}, \hat{t} \epsilon \hat{W}$; then

$$
\langle T \hat{s}, t\rangle=\langle B \Theta \hat{B} \hat{s}, \hat{t}\rangle=[\Theta \hat{B} \hat{s}, \hat{B} \hat{t}]=[\Theta \hat{B} \hat{t}, \hat{B} \hat{s}]^{*}=\langle B \Theta \hat{B} \hat{t}, \hat{s}\rangle^{*}=\langle T \hat{t}, \hat{s}\rangle^{*}
$$

Two systems of vectors $\left\{u_{i}\right\} \subset W,\left\{\hat{u}_{i}\right\} \subset \hat{W}$ will be called biorthogonal if and only if

$$
\left\langle u_{i}, \hat{u}_{j}\right\rangle=\delta_{i j}
$$

Now, assume that the elements $\left\{\hat{u}_{i}\right\} \subset \hat{W}$ have the property

$$
\hat{B}_{\hat{u}_{i}=\hat{\pi}_{i}}
$$

and assume that $\left\{\pi_{i}\right\} \subset \Omega$ is BON to $\left\{\hat{\pi}_{i}\right\} \subset \hat{\Omega}$. Further, assume that

$$
\Theta \hat{\pi}_{i}=\pi_{i}
$$

and let

$$
T \hat{u}_{i}=u_{i}
$$

\footnotetext{
${ }^{2}$ The asterisk in (2) and subsequent expressions will indicate complex conjugate.
} 
Then $\left\{u_{i}\right\}$ is BON to $\left\{\hat{u}_{i}\right\}$. For

$$
\begin{aligned}
\left\langle u_{i}, \hat{u}_{j}\right\rangle & =\left\langle T \hat{u}_{i}, \hat{u}_{j}\right\rangle=\left\langle B \Theta \hat{B}_{\hat{u}_{i}}, \hat{u}_{j}\right\rangle \\
& =\left[\Theta \hat{\pi}_{i}, \hat{\pi}_{j}\right]=\left[\pi_{i}, \hat{\pi}_{j}\right] \\
& =\delta_{i j} .
\end{aligned}
$$

Note that in the calculation (8) it was not necessary to assume that $\Theta$ is self-adjoint; but this will be so for all cases of interest to us.

We will refer to the structure of figure 1 as a "bridge." And we will refer to $B$ and $\hat{B}$ as "bridge" operators. The terminology is not in use, to our knowledge; but it is convenient here.

Operators, Spaces, and Norms. In what follows we shall make the following assumptions:

(a) The operators $B, \hat{B}$, and $\Theta$ are one-to-one and onto, and their domains are the whole spaces $\Omega, \hat{W}$, and $\hat{\Omega}$, respectively.

(b) $B$ and $\hat{B}$ are adjoint, and $\Theta$ is positive definite and self-adjoint, as already indicated.

(c) $\left\{\pi_{i}\right\} \subset \Omega$ and $\left\{\hat{\pi}_{i}\right\} \subset \hat{\Omega}$ are biorthogonal, and $\Theta \hat{\pi}_{i}=\pi_{i}$ for all $i$.

(d) Without loss of generality we may assume that the space $\hat{\Omega}$ is complete in the weak topol$\operatorname{ogy}^{3} \sigma(\hat{\Omega}, \Omega)$, for if it is not, we may consider its abstract completion. We also assume that the set of all finite linear combinations of the $\hat{\pi}_{i}$, designated by $\left\langle\hat{\pi}_{i}\right\rangle$, is dense in $\hat{\Omega}$.

(e) The $\left\{\pi_{i}\right\}$ are linearly independent.

We now define a norm on $\hat{\Omega}$ as follows:

$$
\|\hat{\sigma}\|=[\Theta \hat{\sigma}, \hat{\sigma}]^{1 / 2} .
$$

It can be shown that (9) does indeed define a norm on $\hat{\Omega}$. Further, if

$$
\hat{\sigma}=\sum_{i=0}^{n} \alpha_{i} \hat{\pi}_{i}
$$

then

$$
\begin{aligned}
\|\hat{\sigma}\|^{2} & =\left[\Theta \sum_{i=0}^{n} \alpha_{i} \hat{\pi}_{i}, \sum_{i=0}^{n} \alpha_{i} \hat{\pi}_{i}\right]=\left[\sum_{i=0}^{n} \alpha_{i} \pi_{i}, \sum_{i=0}^{n} \alpha_{i} \hat{\pi}_{i}\right] \\
& =\sum_{i=0}^{n}\left|\alpha_{i}\right|^{2} .
\end{aligned}
$$

We can thus map $\left\{\hat{\pi}_{i}\right\}$ by means of an isometric isomorphism onto the set of all finite sequences with square norm, $E_{\infty}^{2}$. Extending this mapping by continuity we find that $\hat{\Omega}$ is isomorphic to $l^{2}$.

Thus we obtain the following results:

(a) $\hat{\Omega}=\left\{\left.\sum_{i=0}^{\infty} \alpha_{i} \hat{\pi}_{i}\left|\sum_{i=0}^{\infty}\right| \alpha_{i}\right|^{2}<\infty\right\}$

(b) $\hat{\sigma} \epsilon \hat{\Omega} \Rightarrow\|\hat{\sigma}\|^{2}=\sum_{i=0}^{\infty}\left|\alpha_{i}\right|^{2}$.

(c) The set $\left\{\hat{\pi}_{i}\right\}$ is a Schauder basis for $\hat{\Omega}$.

${ }^{3}$ This is the notation used, e.g., by Wilansky, ref. [8], p. 235. 
(d) The spaces $W, \hat{W}$, and $\Omega$ are all isometrically isomorphic to $l^{2}$ and if $\hat{\sigma}=\hat{B} \hat{s}, \sigma=\Theta \hat{\sigma}$, $s=B \sigma=T \hat{s}$, then

$$
\|s\|=\|\hat{s}\|=\langle T \hat{s}, \hat{s}\rangle^{1 / 2}=\|\sigma\|=\|\hat{\sigma}\|=\left\{\sum_{i=0}^{\infty}\left|\alpha_{i}\right|^{2}\right\}^{1 / 2} .
$$

(e) The sets $\left\{u_{i}\right\},\left\{\hat{u}_{i}\right\}$, and $\left\{\pi_{i}\right\}$ identified by (5), (6), and (7) are all Schauder bases for their respective spaces.

While the operator $T$ has been identified by (3) in terms of the $(\Omega, \hat{\Omega},[]$,$) pairing, this operator$ may be defined independently; in this case (10) relates the norm based on $T$ to the norm of the other pairing of the bridge.

This highly structured "bridge," illustrated in figure 1 , is now seen to be an isometric isomorphism between pairings. It enables us to move with confidence in developing and using BON systems.

Kernels and Their Series Representations. In what follows, $W$ and $\hat{W}$ will consist of functions defined on domains $D$ and $\hat{D}$, respectively, of the complex plane. The pairing $\langle$,$\rangle will be defined by$

$$
\langle s, \hat{t}\rangle=\int Z d w s \hat{t}^{*},
$$

where $Z$ is a suitable subset of $D \cap \hat{D}$ and $d w$ will in general include a weight function. Likewise, $\Omega$ and $\hat{\Omega}$ will be spaces of functions defined on domains $\Delta$ and $\hat{\Delta}$, respectively, of the complex plane. The pairing [, ] will be given by

$$
[\sigma, \hat{\tau}]=\int_{\Gamma} d \omega \sigma \hat{\tau}^{*}
$$

where $\Gamma \subseteq \Delta \cap \hat{\Delta}$ and $d \omega$ will similarly include a weight function.

The operators $B$ and $\hat{B}$ are defined using a kernel function $k$, which is integrable on the domain $(D \cup \hat{D}) \times(\Delta \cup \hat{\Delta})$ as follows:

$$
[\hat{B} \hat{t}](\eta)=\int_{Z^{\prime}} d w(z) k(z, \eta) \hat{t}\left(z^{*}\right)
$$

and

$$
[B \sigma](z)=\int_{\Gamma^{\prime}} d \omega(\eta) k\left(z, \eta^{*}\right) \sigma(\eta)
$$

where $Z^{\prime}$ and $\Gamma^{\prime}$ are in $D \cap \hat{D}$ and $\Delta \cap \hat{\Delta}$, respectively. The operator $\Theta: \Omega \rightarrow \Omega$ is of the type

$$
[\hat{\theta \tau}](\eta)=\int_{\Gamma^{\prime \prime}} d \omega\left(\eta^{\prime}\right) \vartheta\left(\eta, \eta^{\prime}\right) \hat{\tau}\left(\eta^{\prime *}\right)
$$

where $\vartheta$, which is integrable on the domain $(\Delta \cup \hat{\Delta})$, is symmetric in $\eta$ and $\eta^{\prime}$, and $\Gamma^{\prime \prime}$ is in $\Delta \cap \hat{\Delta}$.

From (12)-(14) it is clear that the operator $T: \hat{W} \rightarrow W$ has the form

$$
T \hat{s}(z)=\int_{Z} d w(y) t(z, y) \hat{s}\left(y^{*}\right)
$$

where $t(z, y)$ is a kernel function having the form

$$
t(z, y)=\int_{\Gamma^{\prime}} d \omega(\eta) \int_{\Gamma^{\prime \prime}} d \omega\left(\eta^{\prime}\right) k\left(z, \eta^{*}\right) \vartheta\left(\eta, \eta^{\prime}\right) k\left(y, \eta^{*}\right) .
$$


Because the set $\left\{\hat{\pi}_{i}\right\}$ is a basis in $\hat{\Omega}$, we can write, for $z$ fixed in $D$,

$$
k(z, \eta)=\sum_{i} k_{i} \hat{\pi}_{i}(\eta)
$$

The functions $\left\{u_{j}\right\}$ already mentioned are given by

$$
u_{j}=T \hat{u_{j}}=B \pi_{j}=\int_{\Gamma} d \omega(\eta) k\left(z, \eta^{*}\right) \pi_{j}(\eta)
$$

and it is clear from the series representation (17) for $k(z, \eta)$ that

$$
u_{j}(z)=\sum_{i} k_{i}\left[\pi_{j}, \hat{\pi}_{i}\right]=k_{j}
$$

so that $k(z, \eta)$ must be given by

$$
k(z, \eta)=\sum_{i} u_{i}(z) \hat{\pi}_{i}(\eta)
$$

Likewise, from (16) and $\left(17^{\prime}\right)$, the series form for $t(z, y)$ is given by

$$
t(z, y)=\sum_{i} u_{i}(y) B \Theta \hat{\pi}_{i}=\sum_{i} u_{i}(z) u_{i}(y),
$$

where $z, y \in Z$.

\section{The $U_{n}^{k}, \hat{U_{n}^{k}}$ BON System}

Reference BON System. We wish to consider functions $\hat{\pi}_{n}(\eta)$ given by

$$
\hat{\pi}_{n}^{*}(\eta)=\left(\frac{\eta^{2}-1}{\eta^{2}}\right)^{n} \frac{1}{\eta}
$$

together with another set, $\left\{\pi_{n}(\eta)\right\}$, given by

$$
\pi_{n}(\eta)=\left(\frac{\eta^{2}}{\eta^{2}-1}\right)^{n}
$$

where $\eta$ is complex. These two sets of functions are BON for integration along a contour $c$ parallel to the imaginary axis, and crossing the real axis between 0 and $1 / \sqrt{2}$ :

$$
\frac{1}{2 \pi i} \int_{c} d \eta \frac{2}{1-\eta^{2}} \pi_{m}(\eta) \hat{\pi}_{n}^{*}(\eta)=\delta_{n m}
$$

Here $\left\{\frac{2}{1-\eta^{2}}\right\}$ plays the role of a weight function. To see that (21) holds, we note that for $n>m$, the integrand has the form

$$
\sum_{i=0}^{(n-m)-1} a_{i} \eta^{-3-2 i}
$$

which has no residue. Further, for $(m-n)=j \geqslant 1$, the integrand can be written in partial fraction form, 


$$
\frac{-2 \eta^{2 j-1}}{\left(\eta^{2}-1\right)^{j+1}}=\sum_{i=2}^{j+1} c_{i} \frac{1}{(1+\eta)^{i}}-\sum_{i=2}^{j+1} c_{i} \frac{1}{(1-\eta)^{i}}
$$

where the sums begin with $i=2$ because (a) the integrand is odd in $\eta$, and (b) for large $\eta$ the integrand must be of order $\eta^{-3}$. From this form it is obvious that the integral again vanishes; there are no residues at the poles at $\eta= \pm 1$. Lastly, a simple residue calculation establishes the result for $m=n$.

The Spaces $\Omega$ and $\hat{\Omega}$. If, now, in accordance with our discussion in section 2 we define $\hat{\Omega}$ to be the set of all functions $\hat{\Phi}$ satisfying

$$
\hat{\Phi}(\eta)=\sum_{n=0}^{\infty} \alpha_{n} \hat{\pi}_{n}(\eta)
$$

with

$$
\sum_{n=0}^{\infty}\left|\alpha_{n}\right|^{2}<\infty
$$

and $\Omega$ to be the space of all functions $\Phi$ satisfying

$$
\Phi(\eta)=\sum_{n=0}^{\infty} \alpha_{n} \pi_{n}(\eta)
$$

we obtain the following results:

The elements of $\hat{\Omega}$ are essentially power series in $\left(\frac{\eta^{2}-1}{\eta^{2}}\right) *$. The magnitude of this variable will be unity on the equilateral hyperbola given by

$$
\cos 2 \theta_{\eta}=\frac{1}{2|\eta|^{2}}
$$

which is shown in figure 2. For values of $\eta$ in the two regions designated $\mathrm{I}$, to the right and left of the hyperbola, $\left|\frac{\eta^{2}-1}{\eta^{2}}\right|<1$. Thus the series (22) will converge for all $\hat{\Phi}$ and all $\eta$ in regions I; and

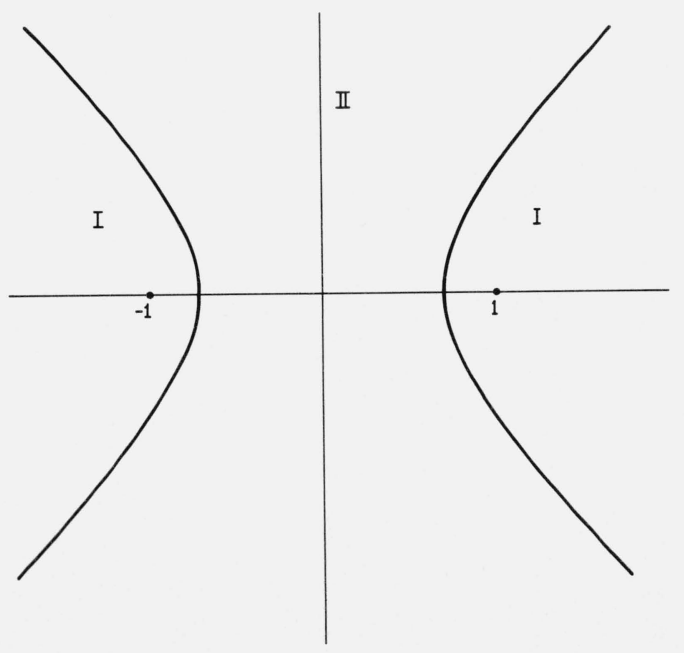

FIGURE 2. The hyperbola intersects the real axis at $\pm 1 / \sqrt{2}$. 
$\hat{\Phi}(\eta)$ can accordingly have no singularity in regions I. Any singularity must either be in region II or on the hyperbola.

From $\left(20^{\prime}\right)$ and $(23)$ it is obvious that the series (24) for $\Phi$ will converge for all $\eta$ in region II.

The transformation $\eta^{\prime}=\sqrt{1-\eta^{* 2}}$ changes $\hat{\Phi}\left(\eta^{\prime}\right)$ into $\frac{1}{\sqrt{1-\eta^{2}}} \Phi(\eta)$ and maps each singularity of $\hat{\Phi}$ in II into a corresponding singularity of $\Phi$ in I. It is possible for $\hat{\Phi}\left(\eta^{\prime}\right)$ to have singularities on the hyperbola. Clearly these are mapped into singularities of $\Phi(\eta)$ at the same locations on the ' hyperbola, since $\left|\frac{\eta^{2}-1}{\eta^{2}}\right|=1$ implies that

$$
\frac{\left(\eta^{\prime *}\right)^{2}-1}{\left(\eta^{\prime *}\right)^{2}}=\frac{\eta^{2}}{\eta^{2}-1}=\frac{\eta^{* 2}-1}{\eta^{* 2}}
$$

Referring back to (19), we identify the BON-system kernel by

$$
\vartheta\left(\eta, \eta^{\prime}\right)=\sum_{n=0}^{\infty} \pi_{n}(\eta) \pi_{n}\left(\eta^{\prime}\right)=\frac{\left(1-\eta^{2}\right)\left(1-\eta^{\prime 2}\right)}{1-\left(\eta^{2}+\eta^{\prime 2}\right)} .
$$

The series converges for $\eta$ and $\eta^{\prime}$ both in region II. From a simple residue calculation we confirm that

$$
\pi_{n}(\eta)=\Theta \hat{\pi}_{n}=\frac{1}{2 \pi i} \int_{c} d \eta^{\prime}\left\{\frac{2}{1-\eta^{\prime 2}}\right\} \frac{\left(1-\eta^{2}\right)\left(1-\eta^{\prime 2}\right)}{1-\left(\eta^{2}+\eta^{\prime 2}\right)} \hat{\pi}_{n}\left(\eta^{\prime *}\right)
$$

If $\hat{\Phi}(\eta)$ is given as in $(22)$, then

$$
[\Theta \hat{\Phi}](\eta)=\Phi(\eta)=\sum_{n=0}^{\infty} \alpha_{n} \Theta \hat{\pi}_{n}=\sum_{n=0}^{\infty} \alpha_{n} \pi_{n}(\eta)
$$

where the kernel of $\Theta$ is given in (26). Likewise, in agreement with (9) and (10) we write

$$
\|\Phi\|=\|\hat{\Phi}\|=\left\{\int_{c} d \eta \frac{2}{1-\eta^{2}} \Phi(\eta) \Phi^{*}(\eta)\right\}^{1 / 2}=\left\{\sum_{n=0}^{\infty}\left|\alpha_{n}\right|^{2}\right\}^{1 / 2} .
$$

It is clear that the sets $\left\{\pi_{n}\right\},\left\{\hat{\pi}_{n}\right\}$ are Schauder bases for $\Omega, \hat{\Omega}$, respectively, and that $\Theta$ is one-to-one onto. The inverse operator, $\Theta^{-1}$ is easy to write down explicitly.

The $\mathrm{U}_{\mathrm{n}}^{\mathrm{k}}$ and $\hat{\mathrm{U}}_{\mathrm{n}}^{\mathrm{k}}$ Functions. We shall now complete the structure outlined in section 2.

For every nonnegative integer $k$ we shall define an operator $B_{k}$ on $\Omega$ generating a sequence of functions which we will call $\left\{U_{n}^{k}\right\}_{n=0}^{\infty}$

$$
U_{n}^{k}=B_{k} \pi_{n}
$$

These operators $B_{k}$. will turn out to have adjoints (in a sense to be defined presently) and we shall then define a system $\left\{\hat{U}_{n}^{k}\right\}_{n=0}^{\infty}$ BON to $\left\{U_{n}^{k}\right\}_{n=0}^{\infty}$ by solving the integral equations

$$
\hat{B}_{k} \hat{U}_{n}^{k}=\hat{\pi}_{n}^{k} \quad n=0,1, \ldots
$$

The spaces $W_{k}$ and $\hat{W}_{k}$ will then be constructed around the $\left\{U_{n}^{k}\right\}$ and $\left\{\hat{U}_{n}^{k}\right\}$ exactly as $\Omega$ and $\hat{\Omega}$ were constructed around the $\left\{\pi_{n}\right\}$ and $\left\{\hat{\pi}_{n}\right\}$. We shall then obtain a BON bridge for every nonnegative integer $k$. 


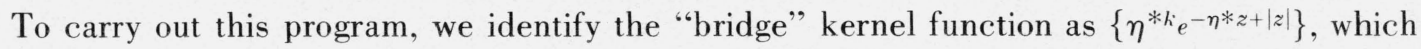
we may also write in the form $e^{|z|}(-)^{k}\left(\frac{\partial^{k}}{\partial z^{k}} e^{-\eta * z}\right)$; and we apply this kernel to the $\pi_{n}(\eta)$. For $z \neq 0$,

$$
\begin{aligned}
U_{n}^{k}(z)=B_{k} \pi_{n}=\frac{1}{2 \pi i} \int_{c_{0}-i \infty}^{c_{0}+i \infty} d \eta \frac{2}{\left(1-\eta^{2}\right)}\left\{e^{|z|}(-)^{k} \frac{\partial^{k}}{\partial z^{k}} e^{-\eta z}\right\} \pi_{n}(\eta) & \\
= & (-)^{k} e^{|z|} \frac{\partial^{k}}{\partial z^{k}} \frac{1}{2 \pi i} \int_{-i \infty}^{i \infty} d \eta e^{-\eta z} \frac{2}{\left(1-\eta^{2}\right)}\left(\frac{\eta^{2}}{\eta^{2}-1}\right)^{n},
\end{aligned}
$$

where $0<c_{0}<1 / \sqrt{2}$. The Fourier integral may be evaluated as follows:

$$
\begin{aligned}
e^{-|z|} U_{n}^{k}(z)=(-)^{n+k}\left(\frac{\partial}{\partial z}\right)^{2 n+k} \frac{1}{2 \pi i} \int_{-i \infty}^{i x} d \eta e^{-\eta z} & \frac{2}{\left(1-\eta^{2}\right)^{n+1}} \\
& =(-)^{n+k} \frac{1}{2^{n} n !} \sqrt{\frac{2}{\pi}}\left(\frac{\partial}{\partial z}\right)^{2 n+k}\left\{|z|^{n+1 / 2} K_{n+1 / 2}(|z|)\right\},
\end{aligned}
$$

where $K_{n+1 / 2}$ is the Hankel function. To this point we have not defined $U_{n}^{k}(z)$ at $z=0$. An examination of the structure of $\left(28^{\prime}\right)$ reveals the reason. The operation $\left(\frac{\partial}{\partial z}\right)^{2 n}$ can be applied to the quantity in braces in $\left(28^{\prime}\right)$ without difficulty, due to an interesting property of the functions $z^{n+1 / 2} K_{n+1 / 2}(z)$ : they lack the flrst $n$ odd powers in the MacLauren expansion. But the next derivative $(k=1)$ not only generates a function odd in $z$, it produces a discontinuity at $z=0$. The second derivative $(k=2)$ produces an even function; and the derivative of the discontinuity generates a delta function at $z=0$. Higher derivatives produce functions of parity $k$ and singular features of the same and higher orders at $z=0$.

The singular features at $z=0$ are not, in general, characteristic of the functions to be represented by the $U_{n}^{k}$; and they contribute to none of the relevant integrals over the $U_{n}^{k}$. Hence we remove them by the simple expedient of defining

$$
U_{n}^{k}(0)=\lim _{z \rightarrow 0^{+}} U_{n}^{k}(z) .
$$

Equation (41) gives these values for $k=0$; for $k=1, U_{n}^{1}(0)=1$ for all $n$.

We now turn to the problem of determining the functions $\hat{U}_{n}^{k}$ which are BON to the $U_{n}^{k}$. To derive these we solve the integral equation $\hat{B}_{k} \hat{U}_{n}^{k}=\hat{\pi}_{n}^{k}$, where $\hat{B}_{k}$ is given by

$$
\frac{1}{2} \int_{-\infty}^{\infty} d z\left\{z^{k} e^{-|z|}\right\}\left\{e^{|z|}\left(\frac{z}{|z|} \eta^{*}\right)^{k} e^{-|z| \eta^{*}}\right\} \hat{U}_{n}^{k}(z)=\hat{\pi}_{n}(\eta) .
$$

This expression simplifies to the complex conjugate form

$$
\int_{0}^{\infty} d z e^{-\eta z}\left\{z^{k} \hat{U}_{n}^{k}(z)\right\}=\left(\frac{\eta^{2}-1}{\eta^{2}}\right)^{n} \frac{1}{\eta^{k+1}} .
$$

From the binomial expansion of the term on the right it is clear that

$$
z^{k} \hat{U}_{n}^{k}(z)=\sum_{i=0}^{n}(-)^{i}\left(\begin{array}{l}
n \\
i
\end{array}\right) \frac{z^{2 i+k}}{(2 i+k) !} .
$$

Finally, we write the BON property of these two sets of functions explicitly to exhibit the pairing integral, and the weight function, clearly: 


$$
\frac{1}{2} \int_{-\infty}^{\infty} d z\left\{z^{k} e^{-|z|}\right\} U_{n}^{k}(z) \hat{U}_{m}^{k}(z)=\int_{0}^{\infty} d z z^{k} e^{-z} U_{n}^{k}(z) \hat{U}_{m}^{k}(z)=\delta_{n m}
$$

The Spaces $\mathrm{W}_{\mathrm{k}}$ and $\hat{\mathrm{W}}_{\mathrm{k}}$. The spaces $W_{k}$ and $\hat{W}_{k}$ can now be constructed as outlined in the previous sections. We define

$$
F_{k}(z) \epsilon W_{k} \Leftrightarrow F_{k}(z)=\sum_{t=0}^{\infty} \alpha_{i} U_{i}^{k} ; \sum\left|\alpha_{i}\right|^{2}<\infty
$$

and

$$
\hat{F}_{k}(z) \epsilon \hat{W}_{k} \Leftrightarrow \hat{F}_{k}(z)=\sum_{t=0}^{\infty} \alpha_{i} \hat{U}_{i}^{k} ; \sum\left|\alpha_{i}\right|^{2}<\infty
$$

It is clear that the elements $F_{k}(z)$ of the space $W_{k}$ will consist of the functions $B_{k} \Phi$, where $\Phi \epsilon \Omega$. That is, for $z \neq 0$,

$$
F_{k}(z)=(-)^{k} e^{|z|}\left(\frac{\partial}{\partial z}\right)^{k} \frac{1}{2 \pi i} \int_{-i \infty}^{i \infty} d \eta e^{-\eta z} \frac{2}{1-\eta^{2}} \Phi(\eta)
$$

Note that $F_{k}(z)$ has parity $(-)^{k}$. If $\lim _{z \rightarrow 0^{+}} F_{k}(z)$ exists, we define $F_{k}(0)$ to have this value.

The function $F_{k}(z)$, which is given by

$$
F_{k}(z)=\sum_{n=0}^{\infty} \alpha_{n} U_{n}^{k}(z)
$$

exists and is bounded for all $k$, all $\Phi \epsilon \Omega$ and all $z \neq 0$. This is partly because of (23) and partly because of the fact yet to be shown that

$$
\left|U_{n}^{k}(z)\right|=\mathrm{O}\left(n^{-1 / 2}\right) .
$$

The inverse operator, $B_{k}^{-1}$, is easy to write down and can be useful in identifying elements of $W_{k}$ :

$$
B_{k}^{-1} F_{k}(z)=\int_{-\infty}^{\infty} d z\left\{\frac{\left(1-\eta^{2}\right)}{2 \eta^{k}} e^{\eta z}\right\} e^{-|z|} F_{k}(z)=\Phi(\eta)
$$

We recall that $\Phi(\eta)$ has singularities in region I, and that the power series which comprise the elements of $\Omega$ can quite generally represent functions with singularities in this region or on the hyperbola. Accordingly, we identify the elements of $W_{k}$ by parity, by the feasibility of performing $k$ integrations by parts on $e^{-|z|} F_{k}(z)$, and by the location of the singularities of the Fourier transform after this modification of the integrand.

To identify the space $\hat{W}_{k}$, we first observe that we can write the operator $\hat{B}_{k}$ in the form of a Laplace transformation. Referring to (30), we should have, for each $\hat{F}_{k} \in W_{k}$,

$$
\int_{0}^{\infty} d z e^{-\eta^{*} z} z^{k} \hat{F}_{k}(z)=\frac{1}{\eta^{* k}} \hat{\Phi}(\eta)
$$

or, eliminating the factor $\eta^{* k}$ by successive integrations by parts,

$$
\int_{0}^{\infty} d z e^{-\eta^{*} z}\left(\frac{d}{d z}\right)^{k} z^{k} \hat{F}_{k}(z)=\hat{\Phi}(\eta)
$$


If the function $\hat{F}_{k}$ has even parity, and if

$$
\left(\frac{d}{d z}\right)^{k} z^{k} \hat{F}_{k}(z)
$$

has a Laplace transform whose singularities are confined to region II, then surely $\hat{F}_{k} \epsilon \hat{W}_{k}$. Further, since the operators $\hat{B}_{k}, \theta$, and $B_{k}$ are all clearly one-to-one, the function $F_{k}=\left(B_{k} \Theta \hat{B}_{k}\right) \hat{F}_{k}$ is then an element of $W_{k}$.

The Norm and BON-System Kernel for $\mathrm{W}_{\mathrm{k}}$ and $\hat{W}_{\mathrm{k}}$. We define a norm on both $W_{k}$ and $\hat{W}_{k}$ as already indicated:

$$
\left\|F_{k}\right\|=\left\|F_{k}\right\|=\left\{\int_{0}^{\infty} d z\left\{z^{k} e^{-z}\right\} F_{k}(z) \hat{F}_{k}^{*}(z)\right\}^{1 / 2}=\left\{\sum_{n=0}^{\infty}\left|\alpha_{n}\right|^{2}\right\}^{1 / 2}
$$

It is obvious that the $\left\{U_{n}^{k}\right\}$ and $\left\{\hat{U}_{n}^{k}\right\}$ constitute Schauder bases for $W_{k}$ and $\hat{W}_{k}$, respectively.

The operator $T_{k}$, with the property $T_{k} \hat{F}_{k}=F_{k}$, together with the BON-system kernel $t_{k}(z, y)$ are easily identified. If $z \neq 0$, we have

$$
\begin{aligned}
\left(B_{k} \Theta \hat{B}_{k}\right) \hat{F}_{k}=\{( & \left.-)^{k} e^{|z|}\left(\frac{\partial}{\partial z}\right)^{k} \frac{1}{2 \pi i} \int_{-i \infty}^{i \infty} d \eta e^{-\eta z} \frac{2}{1-\eta^{2}}\right\}\left\{\frac{1}{2 \pi i} \int_{c} d \eta^{\prime} \frac{2}{1-\eta^{\prime 2}} \frac{\left(1-\eta^{2}\right)\left(1-\eta^{\prime 2}\right)}{1-\left(\eta^{2}+\eta^{\prime 2}\right)}\right\} \\
& \times\left\{\int_{0}^{\infty} d y y^{k}(-)^{k}\left(\frac{\partial}{\partial y}\right)^{k} e^{-\eta^{\prime} y}\right\} \hat{F}_{k}(y), \\
= & \int_{0}^{\infty} d y\left(y^{k} e^{-y}\right)\left\{e^{|z|+|y|}\left(\frac{\partial^{2}}{\partial z \partial y}\right)^{k} \frac{1}{2 \pi i} \int_{-i \infty}^{i \infty} d \eta e^{-\eta z-y \sqrt{1-\eta^{2}}} \frac{2}{\sqrt{1-\eta^{2}}}\right\} \hat{F}_{k}(y), \\
& =\int_{0}^{\infty} d y\left(y^{k} e^{-y}\right)\left\{e^{|z|+|y|}\left(\frac{\partial^{2}}{\partial z \partial y}\right)^{k} \frac{2}{\pi} K_{0}\left(\sqrt{z^{2}+y^{2}}\right)\right\} \hat{F}_{k}(y) .
\end{aligned}
$$

From this it is clear that for $z \neq 0$,

$$
T_{k} \hat{F}_{k}=\frac{1}{2} \int_{-\infty}^{\infty} d y\left(y^{k} e^{-|y|}\right)\left\{e^{|z|+|y|}\left(\frac{\partial^{2}}{\partial z \partial y}\right)^{k} \frac{2}{\pi} K_{0}\left(\sqrt{z^{2}+y^{2}}\right)\right\} \hat{F}_{k}(y)
$$

where $K_{0}$ is the Hankel function, and the term in braces is the kernel function.

The behavior of the kernel for higher $k$ values can to some extent be inferred from the $k=1$ case:

$$
\left(\frac{\partial^{2}}{\partial y \partial z}\right) \frac{2}{\pi} K_{0}\left(\sqrt{z^{2}+y^{2}}\right)=\frac{2}{\pi} \frac{y z}{\left(y^{2}+z^{2}\right)} K_{2}\left(\sqrt{y^{2}+z^{2}}\right)
$$

which, for small values of the aroument, is proportional to $y z /\left(y^{2}+z^{2}\right)^{2}$.

\section{Properties and Expansions}

Recursion Relations which Connect Systems with Different k Values. If we differentiate (28') with respect to $z$ we can show that for $z \neq 0$,

$$
\frac{d}{d z}\left\{e^{-|z|} U_{n}^{k}(z)\right\}=-e^{-|z|} U_{n}^{k+1}(z)
$$

Analogously, inspection of $\left(30^{\prime}\right)$ shows that

$$
\int_{0}^{z} d z^{\prime} z^{\prime} \hat{U}_{n}^{k}\left(z^{\prime}\right)=z^{k+1} \hat{U}_{n}^{k+1}(z)
$$


A more elaborate recursion relation, which likewise connects systems with different values of $k$, can be obtained from (28) by integration by parts. We write ${ }^{4}$

$$
z^{k} e^{-|z|} U_{n}^{k}(z)=\frac{(i z)^{k}}{\pi} \int_{-\infty}^{\infty} d s e^{-i s z}\left\{\frac{s^{2 n+k}}{\left(1+s^{2}\right)^{n+1}}\right\}
$$

Integration by parts $k$ times gives

$$
z^{k} e^{-|z|} U_{n}^{k}(z)=\frac{1}{\pi} \int_{-\infty}^{\infty} d s e^{-i s z}\left(\frac{d}{d s}\right)^{k}\left\{\frac{s^{2 n+k}}{\left(1+s^{2}\right)^{n+1}}\right\} .
$$

Carrying out one of the indicated differentiations, we obtain

$$
\begin{aligned}
z^{k} e^{-|z|} U_{n}^{k}(z)=\frac{1}{\pi} \int_{-\infty}^{\infty} d s e^{-i s z}\left(\frac{d}{d s}\right)^{k-1} & \left(k \frac{s^{2 n+(k-1)}}{\left(1+s^{2}\right)^{n+1}}-2 \frac{s^{2(n+1)+(k-1)}}{\left(1+s^{2}\right)^{n+2}}\right. \\
+ & \left.2 n\left\{\frac{s^{2 n+(k-1)}}{\left(1+s^{2}\right)^{n+1}}-\frac{s^{2(n+1)+(k-1)}}{\left(1+s^{2}\right)^{n+2}}\right\}\right) \\
& =z^{k-1} e^{-|z|}\left\{k U_{n}^{k-1}(z)-2 U_{n+1}^{k-1}(z)+2 n\left[U_{n}^{k-1}(z)-U_{n+1}^{k-1}(z)\right]\right\}
\end{aligned}
$$

Some recursion relations which connect different functions for the same value of $k$ will be included in a report to follow.

The Trend of the $\mathrm{U}_{\mathrm{n}}^{\mathrm{k}}$ for Large Values of $\mathrm{n}$. We now wish to examine $\lim _{n \rightarrow \infty} U_{n}^{k}(z)$. We begin by considering the case $k=0$, for which we write

$$
\begin{gathered}
\left|e^{-|z|} U_{n}^{0}(z)\right|=\left|\frac{2}{\pi} \int_{0}^{\infty} d s \cos s z\left(\frac{s^{2}}{1+s^{2}}\right)^{n} \frac{1}{1+s^{2}}\right| \\
\leqslant \frac{2}{\pi} \int_{0}^{\infty} d s\left(\frac{s^{2}}{1+s^{2}}\right)^{n} \frac{1}{1+s^{2}} \leqslant \frac{1}{\sqrt{\pi}} \frac{\Gamma\left(n+\frac{1}{2}\right)}{\Gamma(n+1)}=U_{n}^{0}(0) .
\end{gathered}
$$

Next, we change the variable of integration in (40) to $u=s / \sqrt{n+1}$ and rewrite in the following form:

$$
z^{k} e^{-|z|} U_{n}^{k}(z)=\frac{1}{\sqrt{n+1}} \frac{2}{\pi} \int_{0}^{\infty} d u \cos (u z \sqrt{n+1})\left(\frac{d}{d u}\right) k_{u} k-2\left\{e^{-1 / u^{2}}+R(n, u)\right\}
$$

where $R(n, u)=\exp \left\{-(n+1) \log \left[1+u^{-2}(n+1)^{-1}\right]\right\}-\exp \left(-1 / u^{2}\right)$. The $R(n, u)$ gives a correction with trend approaching proportionality to $(n+1)^{-3 / 2}$ for large $n$.

We now observe that the main part of the integrand has the form

$$
\left(\frac{d}{d u}\right)^{k}\left(u^{k-2} e^{-1 / u^{2}}\right)=e^{-1 / u^{2}} \sum_{i=0}^{I_{k}} a_{k i} u^{-2-2 i},
$$

where $I_{k}$ is finite and $a_{k 0}$ is zero for $k>1$. Then

$$
\begin{array}{r}
\left|\frac{2}{\pi} \int_{0}^{\infty} d u \cos (u z \sqrt{n+1})\left(\frac{d}{d u}\right)^{k}\left(u^{k-2} e^{-1 / u^{2}}\right)\right| \leqslant \frac{2}{\pi} \int_{0}^{\infty} d u|\cos (u z \sqrt{n+1})|\left|\sum_{i=0}^{I_{k}} a_{k i} u^{-2-2 i} e^{-1 / u^{2}}\right| \\
<\sum_{i=0}^{I_{k}}\left|a_{k i}\right| \frac{2}{\pi} \int_{0}^{\infty} d u u^{-2-2 i} e^{-1 / u^{2}}<C_{k},
\end{array}
$$

where $C_{k}$ is a positive constant which, for $k=0$, has the value $\frac{1}{2} \sqrt{\pi}$, in agreement with (41).

${ }^{4}$ This integrand has a nonconvergent component which contributes only to the (irrelevant) singularities at $z=0$, and which does not invalidate the results of this argument. The integral on the right of $(40)$ lacks this component. 
We conclude that

$$
\left|U_{n}^{k}(z)\right|<\frac{C_{k}}{\sqrt{n+1}}|z|^{-k} e^{|z|}
$$

and it is evident from this that for large values of $n$, for $z \neq 0$, and for any $k$,

$$
U_{n}^{k}(z)=\mathrm{O}\left(n^{-1 / 2}\right)
$$

Generating Functions. Useful generating functions for both $U_{n}^{k}$ and $\hat{U}_{n}^{k}$ are easily derived. First, consider the sum

$$
\sum_{n=0}^{\infty} u^{n}\left\{\eta^{k}\left(\frac{-\eta^{2}}{1-\eta^{2}}\right)^{n} \frac{2}{1-\eta^{2}}\right\}=\frac{2 \eta^{k}}{1-(1-u) \eta^{2}}
$$

The singularities for this function are located at $\eta=\frac{ \pm 1}{\sqrt{1-u}}$, and will be located in regions I for $|u|<1$. Fourier inversion of both sides gives (for $z \neq 0$ )

$$
\left(-\frac{\partial}{\partial z}\right)^{k} \frac{1}{\sqrt{1-u}} e^{-\frac{|z|}{\sqrt{1-u}}}=\sum_{n=0}^{\infty} u^{n} e^{-|z|} U_{n}^{k}(z) .
$$

This may be written,

$$
\left(\frac{z}{|z|}\right)^{k}\left(\frac{1}{\sqrt{1-u}}\right)^{k+1} e^{-\frac{|z|}{\sqrt{1-u}}}=\sum_{n=0}^{\infty} u^{n} e^{-|z|} U_{n}^{k}(z)
$$

and it is clear that for $|u|<1$, this function is an element of $W_{k}$.

The convergence of $\left(45^{\prime}\right)$ for $|u|=1$ is important for later developments; and we establish this next. Note that the function in $\left(43^{\prime}\right)$ is not in $W_{k}$ if $|u|=1$. For $z \neq 0$ we write

$$
\begin{aligned}
\sum_{n=0}^{N} u^{n} e^{-|z|} U_{n}^{k}(z) & =\frac{1}{2 \pi i} \int_{-i \infty}^{i \infty} d \eta e^{-\eta z} \frac{2 \eta^{k}}{1-\eta^{2}} \sum_{n=0}^{N} u^{n}\left(\frac{\eta^{2}}{\eta^{2}-1}\right)^{n} \\
& =\frac{1}{2 \pi i} \int_{-i \infty}^{i \infty} d \eta e^{-\eta z} \frac{2 \eta^{k}}{1-\eta^{2}(1-u)}\left\{1-u^{N+1}\left(\frac{\eta^{2}}{\eta^{2}-1}\right)^{N+1}\right\} .
\end{aligned}
$$

The first term in braces gives the result of $\left(45^{\prime}\right)$. The nature of the second term becomes clear if we write the factor outside the braces as follows:

$$
\frac{2 \eta^{k}}{1-\eta^{2}(1-u)}=\frac{1}{1-u}\left[\eta^{k}-\frac{u \eta^{k}}{1-\eta^{2}(1-u)}\right] \frac{2}{1-\eta^{2}}
$$

The term in brackets reduces to a finite sum of terms $\eta^{k-2 i} /(1-u)^{i}$, plus a proper fraction. The Fourier transformation thus yields a finite sum $\sum_{i} e^{-|z|}(1-u)^{-i} U_{N+1}^{k-2 i}(z)$, plus a convolution over $U_{N+1}^{0}(z)$ or $U_{N+1}^{1}(z)$. All of these terms tend to zero as $N$ becomes large, because of (43). Hence (45') holds, for $z \neq 0$ and for $|u|=1$, so long as $u \neq 1$. 
To obtain a generating function for the $\hat{U}_{n}^{k}$, we consider the sum

$$
\begin{aligned}
\sum_{k=0}^{\infty}(-t)^{k} \sum_{n=0}^{\infty} u^{n}\left\{\left(1-\frac{1}{\eta^{2}}\right)^{n} \frac{1}{\eta^{k+1}}\right\}=\frac{1}{\eta+t} \frac{1}{1-u\left(1-1 / \eta^{2}\right)} & \\
= & \frac{1}{t^{2}(1-u)+u}\left\{\frac{t^{2}}{\eta+t}+\frac{u(\eta-t)}{u+\eta^{2}(1-u)}\right\}
\end{aligned}
$$

It is obvious that for $|u|<1$, and $|t|<\frac{1}{\sqrt{2}}$ ( $t$ real), the singularities of this function all lie in region II. Laplace inversion gives the result ${ }^{5}$

$$
\begin{array}{r}
\frac{1}{t^{2}(1-u)+u}\left\{t^{2} e^{-t|z|}+\frac{u}{1-u} \cos z \sqrt{\frac{u}{1-u}}-t \sqrt{\frac{u}{1-u}} \sin z \sqrt{\frac{u}{1-u}}\right\} \\
=\sum_{k=0}^{\infty}(-t)^{k} \sum_{n=0}^{\infty} u^{n} z^{k} \hat{U}_{n}^{k}(z) .
\end{array}
$$

As special cases we obtain (see footnote 5)

$$
\begin{gathered}
\frac{1}{1-u} \cos z \sqrt{\frac{u}{1-u}}=\sum_{n=0}^{\infty} u^{n} \hat{U}_{n}^{0}(z) \\
\frac{1}{1-u} \sin z \sqrt{\frac{u}{1-u}}=\sum_{n=0}^{\infty} \sqrt{\frac{u}{1-u}} u^{n} z \hat{U}_{n}^{1}(z)
\end{gathered}
$$

and these functions, for $|u|<1$, are elements of $\hat{W}_{0}$ and $\hat{W}_{1}$, respectively. In combination, (48) and $\left(48^{\prime}\right)$ give

$$
\frac{1}{1-u} e^{i z \sqrt{\frac{u}{1-u}}}=\sum_{n=0}^{\infty} u^{n}\left\{\hat{U}_{n}^{0}(z)+i \sqrt{\frac{u}{1-u}} z \hat{U}_{n}^{1}(z)\right\} .
$$

The $\mathrm{U}_{\mathrm{n}}^{\mathrm{k}}$ Polynomials and the Laguerre Polynomials. If in eq $\left(45^{\prime}\right)$ we write $u=(p-1) / p$, the convergence of the series will be maintained for Re $p \geqslant(1 / 2)$. Term-by-term Laplace inversion of this expression multiplied by $p^{-1}$ is possible, and yields the result

$$
\sum_{n=0}^{\infty} L_{n}(t) e^{-|z|} U_{n}^{k}(z)=\frac{1}{\sqrt{\pi t}} \frac{1}{(2 t)^{k / 2}} e^{-z^{2} / 4 t} H e_{k}\left(\frac{z}{\sqrt{2 t}}\right),
$$

where $L_{n}$ and $H e_{k}$ are the $n$th Laguerre and $k$ th Hermite polynomials, respectively.

Note that (49) is the kernel for a transformation $B_{k}$ which links the spaces $W_{k}$ and $\hat{W}_{k}$ with the Hilbert space spanned by the Laguerre polynomials.

The $\mathrm{U}_{\mathrm{n}}^{\mathrm{k}}$ Polynomials and Tchebycheff Polynomials. In precisely the same fashion we can establish bridges with other Hilbert spaces. In particular, let us write $u=e^{i \theta}$ in $\left(45^{\prime}\right)$, which is permissible in view of the convergence argument of section 12. The real part of the result can be written in the following form, which defines a function $\kappa_{k}(z, \theta)$ :

${ }^{5}$ These results, in a different series form, have been given by Preiser (ref. [4]). 


$$
\begin{aligned}
\kappa_{k}(z, \theta) & =\sum_{n=0}^{\infty} \cos n \theta e^{-|z|} U_{n}^{k}(z) \\
= & \frac{(|z| / z)^{k}}{(\sqrt{2 \sin \theta 2)}} k+1 \operatorname{Re} \exp \left\{-\frac{|z|}{\sqrt{2 \sin \theta / 2}} e^{i(\pi-\theta) / 4}+i(k+1)(\pi-\theta) / 4\right\} .
\end{aligned}
$$

A variant of this result is perhaps more useful in applications. Recalling that $\cos (n \theta)$ is the Tchebycheff polynomial $T(\cos \theta)$, we write $x=\left(\frac{1+\cos \theta}{2}\right)=\cos ^{2}(\theta / 2)$ to obtain

$$
T_{n}(2 x-1)=\cos n\left[2 \cos ^{-1} \sqrt{x}\right] .
$$

This polynomial system is actually one of the Jacobi family, and $\sqrt{\epsilon_{n}} T_{n}(2 x-1)$ is orthonormal on the interval $0<x<1$ with respect to the weight function $\frac{1}{\pi \sqrt{x(1-x)}}$. Writing

$$
u=\exp i\left[2 \cos ^{-1} \sqrt{x}\right]
$$

in $\left(45^{\prime}\right)$, we obtain

$$
\kappa_{k}\left(z, 2 \cos ^{-1} \sqrt{x}\right)=\sum_{n=0}^{\infty} T_{n}(2 x-1) e^{-|z|} U_{n}^{k}(z) .
$$

This transformation kernel links $W_{k}$ and $\hat{W}_{k}$ with the space spanned by these Jacobi polynomials.

The $\mathrm{U}_{\mathrm{n}}^{\mathrm{k}}$ 's and the Legendre Polynomials. A bridge for the Legendre polynomials is likewise useful and easy to write down. A generating function for the Legendre polynomials is

$$
\frac{1}{\left\{1-2 s \cos \theta+s^{2}\right)^{1 / 2}}=\sum_{n=0}^{\infty} s^{n} P_{n}(\cos \theta)
$$

The expression on the left has singularities for $|s|=1$, so that the series converges uniformly for $|s|<a<1$, where $a$ is constant.

We write $\eta^{2} /\left(\eta^{2}-1\right)$ in place of $s$ in (54) to obtain

$$
\frac{2}{\left(1-\eta^{2}\right)} \frac{1}{\left\{1-2 \cos \theta\left(\frac{\eta^{2}}{\eta^{2}-1}\right)+\left(\frac{\eta^{2}}{\eta^{2}-1}\right)^{2}\right\}^{1 / 2}}=\frac{2}{\left(1-\eta^{2}\right)} \sum_{n=0}^{\infty} \pi_{n}(\eta) P_{n}(\cos \theta) .
$$
For $\cos \theta \neq 1$, this function is in $\Omega_{-}\left(\right.$because $\sum_{n=0}^{\infty} P_{n}^{2}(\cos \theta)$ converges) and we calculate its
Fourier inverse to obtain

$$
\sum_{n=0}^{\infty} e^{-|z|} U_{n}^{0}(z) P_{n}(\cos \theta)=\frac{2}{\pi} \int_{0}^{\infty} \frac{d \alpha}{1+\alpha^{2}} \frac{\cos \alpha z}{\sqrt{1-2\left(\frac{\alpha^{2}}{1+\alpha^{2}}\right) \cos \theta+\left(\frac{\alpha^{2}}{1+\alpha^{2}}\right)^{2}}} .
$$

Unlike the preceding kernels, this integral does not appear to yield an elementary quadrature in terms of known functions.

Existence of Functions with Zero Alternate Moments. One question which is implicitly an- 
swered by the preceding analysis has to do with the existence of functions $F(z)$, defined on $0 \leqslant \mathrm{z} \leqslant \infty$, which are not identically zero but whose alternate moments $F_{n}$ are all zero beyond some value $n=k-2$.

Let an even function $F(z)$ have a Fourier transform $\Phi(\eta)$ with singularities only at $\eta= \pm \eta_{0}$, Re $\eta_{0} \neq 0$. Then one can choose a number $a$ such that $F(a z)$ has transform singularities in region I, and hence can be represented by a $U_{n}^{0}$ series. If all even moments on $0 \leqslant z \leqslant \infty$ of $F(z)$ should be zero, all coefficients of this series must be zero also, so that $F(a z)$ must be identically zero. Thus if $F(z)$ is not zero identically, these even moments cannot all vanish.

This argument can be extended to odd functions and their odd moments, and to functions of either parity which might have alternate moments zero beyond $n=(k-2)$, by use of expansions with higher values of $k$. The argument then applies to some component of any function whose Fourier transform has a singularity off the imaginary axis, and we conclude that only functions with singularities restricted to the imaginary axis, or at $\infty$, can have zero moments beyond some finite value $n$ for the moment index.

Gamma Ray and Neutron Penetration Distributions. A series of investigations of the deep penetration trends of these distributions has shown that the Fourier transform can have singularities of the following types

$$
\frac{1}{\left(\mu_{0}-p\right)^{\xi}}, \quad e^{-\frac{c}{\mu-p}}, \quad e^{-\frac{\sqrt{c}}{\sqrt{\mu_{m}-p}}},
$$

in the important cases of isotropic or plane monodirectional sources. Here, $\mu_{0}, \mu, \mu_{m}, c$, and $\xi$ are all constants. By measuring distances in units of $\mu_{0}^{-1}, \mu^{-1}$, or $\mu_{m}^{-1}$, all three singularities may be located at $p=1$; and in each case the only other possible singularities are located on the real axis, with $p>1$.

For cases of plane, slant sources, the location and nature of the singularities is not so completely specifiable. If distances are measured in $\mu_{m}^{-1}$, where $\mu_{m}$ is the smallest attenuation coefficient, we can say that the singularities are again on the real axis, with $p \geqslant 1$; but we cannot specify them in greater detail.

In all these cases, the even or odd component distribution functions belong to $W_{k}$ according to the value of $(-)^{k}$, so that $U_{n}^{k}(z)$ series representations should have square summable coefficients, and should converge to the distribution. Most approximations of this type have implicitly used the norms of the $U_{n}^{k}$ systems; since convergence of the polynomial coefficients has been used as a criterion of validity of the approximation.

\section{References}

[1] Spencer, L. V., and U. Fano, Penetration and diffusion of x-rays. Calculation of spatial distributions by polynomial expansion. J. Res. NBS 46, 446 (1951) RP2213.

[2] Goldstein, H., and J. E. Wilkins, Jr., Calculations of the Penetration of Gamma Rays, USAEC Report, NYO-3075 (1954).

[3] Fano, U., L. V. Spencer, and M. J. Berger, Penetration and diffusion of x-rays, Handbuch der Physik 38, 660 (1959) (Springer-Verlag, Berlin).

[4] Preiser, Stanley, An investigation of biorthogonal polynomials derivable from ordinary differential equations of the third order, p. 38, J. Math. Anal. \& Appl. 4, No. 1 (Feb. 1962).

[5] Spencer, L. V., Penetration and diffusion of x-rays: Calculation of spatial distributions by semiasymptotic methods, Phys. Rev. 88, 793 (1952).

[6] Spencer, L. V., Energy dissipation by fast electrons, NBS Mono. 1 (1959).

[7] Kelley, J. L., and I. Namioka, Linear Topological Spaces (D. Van Nostrand Co., Inc., New York, 1963).

[8] Wilansky, Albert, Functional Analysis (Blaisdell Publishing Co., New York, 1964).

(Paper 71B4-244) 